\section{The Moving Finger Writes: Carbon Nanotubes as AFM Probe Tips}

\author{
Katerina Moloni \\ nPoint, Inc.
}

\section{katerina.moloni@npoint.com}

After carbon nanotubes (CNT) were discovered in 1991 [1], many applications have been proposed that utilize their extraordinary electrical and mechanical properties. One application is as tips for scanning probe microscopy where CNTs offer several advantages including high resolution and the capability to image deep, narrow structures $[2,3]$. A recent study of CNT scanning probes for atomic force microscopy (AFM) in semiconductor surface science concluded that an AFM with CNT tips has immense potential as a surface characterization tool in integrated circuit manufacture [4]. Previously researchers had to construct their own CNT probes, but recently CNT AFM probes have become commercially available.

Carbon nanotubes (sometimes called buckytubes) are closed seamless shells of graphitic carbon typically one to tens of nanometers in diameter and several micrometers long. The structure of a closed-dome single-walled nanotube is illustrated in Figure 1. Carbon nanotube probe tips offer several advantages. CNTs can be as small as $1-2 \mathrm{~nm}$ in diameter and tens of $\mathrm{nm}$ long, provide high image resolution, and can accurately measure extremely highaspect-ratio topography. CNTs are 100 times stronger than steel yet are quite flexible and do not deform biological surfaces. In addition, the extreme hardness of the CNT tip provides wear resistance and greater scanning life and their flexibility makes them resistant to damage from probe tip crashes.

CNT probe tips for atomic force microscopy (AFM) consist of single-walled or multi-wall nanotubes (MWNTs) mounted onto AFM probes, typically attached to the ends of etched silicon tips on silicon cantilevers. MWNT tips are now commercially available. CNTs offer unique advantages over standard AFM tips in applications ranging from probing the interiors of biological cells to advanced integrated circuit manufacture. This article presents examples

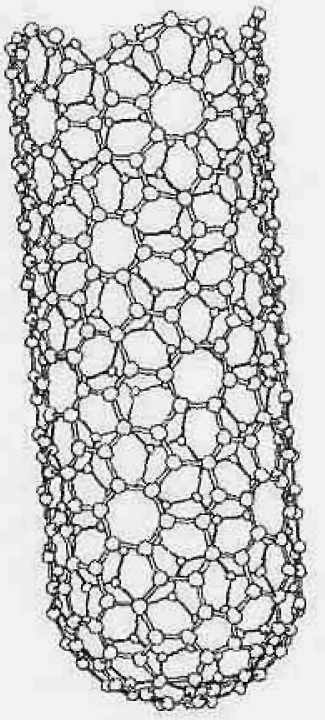

Figure 1. Diagram of the structure of a singlewalled carbon nanotube. of applications of CNT probes and pointers on matching the probe type to the application.

\section{CNT Tip Properties}

Carbon nanotubes have been used as tips for AFM and scanning tunneling microscopy (STM) to take advantage of their nanoscale size, electrical conductivity, wear resistance, and mechanical strength. An example of the enhanced resolution offered by a CNT tip is shown by the images of a photoresist surface in Figure 2. The large length/width aspect ratio plus the small ciameter permits imaging of nanometerscale, high-aspect ratio features, as found on integrated circuits and microelectromechanical systems (MEMS) and structures (Figure 3).

Most conventional AFM tips are silicon nitride or silicon. The radius and shape of these tips vary from one to the other and must be characterized, i.e., their shape accurately determined, to ensure accurate imaging. These tips are subject to wear after repeated scans. In general, the sharper the tip, the better the image resolution, and a worn tip will degrade the image.
Commercially available carbon nanotube (CNT) prove ups for atomic force microscopes (AFMs) typically consist of multiwall nanotubes (MWNT) mounted onto standard AFM cantilever probes, usually tapping mode etched silicon probes. The tips can be mounted on either standard silicon probes or silicon probes coated with platinum/iridium ( $\mathrm{Pt} / \mathrm{Ir}$ ). The metallic coating enhances electrical conductivity and the reflectivity of the laser beam that is used to detect the probe deflection during scanning. As previously indicated, CNT tips offer a much greater scanning life than standard probes and have the ability to achieve high resolution and measure high-aspect ratio features. CNT probes enhance performance in intermittent and non-contact modes but are not recommended for contact mode. Two applications where CNT AFM tips have unique advantages are advanced semiconductor manufacturing and biological research.

\section{Advanced Semiconductor Manufacturing}

\section{Surface Characterization}

The AFM offers a simple, nondestructive method to characterize advanced semiconductor structures in the nanoscale realm. In contrast to techniques such as scanning electron microscopy or transmission electron microscopy, AFMs do not require a special (usually destruc-

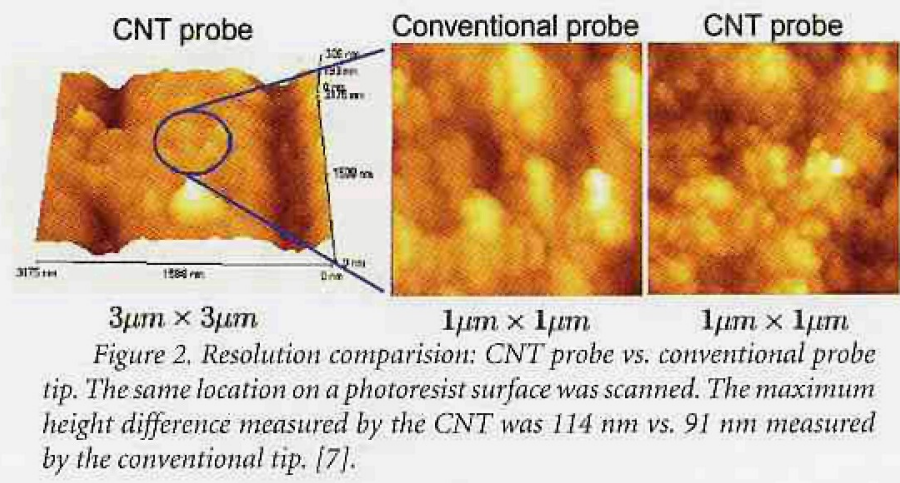

tive) sample preparation or a vacuum environment. AFM has been deemed the best solution for examining surface morphology and uniformity of coverage of non-conducting ultra-thin films. CNT tips extend the capability of conventional AFMs, providing wear resistance, high resolution, and the ability to image very small, high-aspect ratio features. Nguyen, et al. used single-walled nanotube (SWNT) probes in imaging silicon nitride, iridium, and gold films on mica substrates and MWNT probes for metrology of 300-nm-thick photoresist patterns on a silicon substrate [4]. They found that although a single-wall CNT gave better resolution than the larger-diameter MWNT, the MWN'T is much stiffer and can be longer (up to several $\mu \mathrm{m}$ ) without exhibiting thermal vibration. The MWNT could trace deep features, which is important for accurate measurement of the critical dimensions (both widths and thicknesses) of lines and spaces in semiconductor masking layers and patterned structures. To be useful, the tip shape must remain stable during extended use. They found that after con-
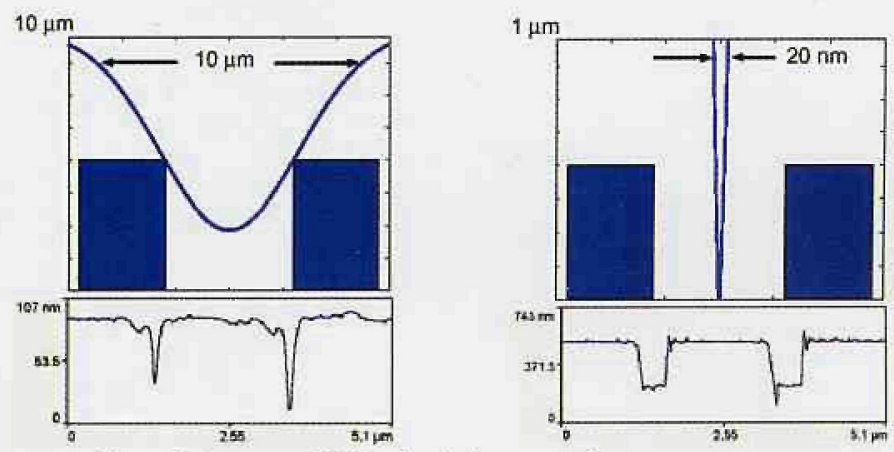

Figure 3. A narrow CNT probe tip improves the measurement accuracy of high-aspect ratio features such as deep, narrow trenches. [8]. 
ADDING ZOOM
YOUR AFM WO
HUR R ALY

If you have been looking to add new application capabilities to your existing AFM or would like to reduce the time spent acquiring the images you need, nPoint has a solution. The $\mathrm{iC}^{\mathrm{c}}$ Closed-loop AFM Upgrade Kit adds an unprecedented level of precision and repeatability to your Dimension $3100^{\circ}$ or other large-samplestage AFMs. The iC ${ }^{\circ}$ Closed-loop AFM Upgrade Kit provides sub-nanometer accuracy and exceptional scan linearity in an easy-toinstall kit. The iC Kit can be used as a substitute for the scanning capabilities of your AFM tube scanner, used as a sample positioner, or used outside the AFM environment for other experiments. The iC Kit's functionality combined with its price provide a previously unavailable level of value in AFM instrumentation.
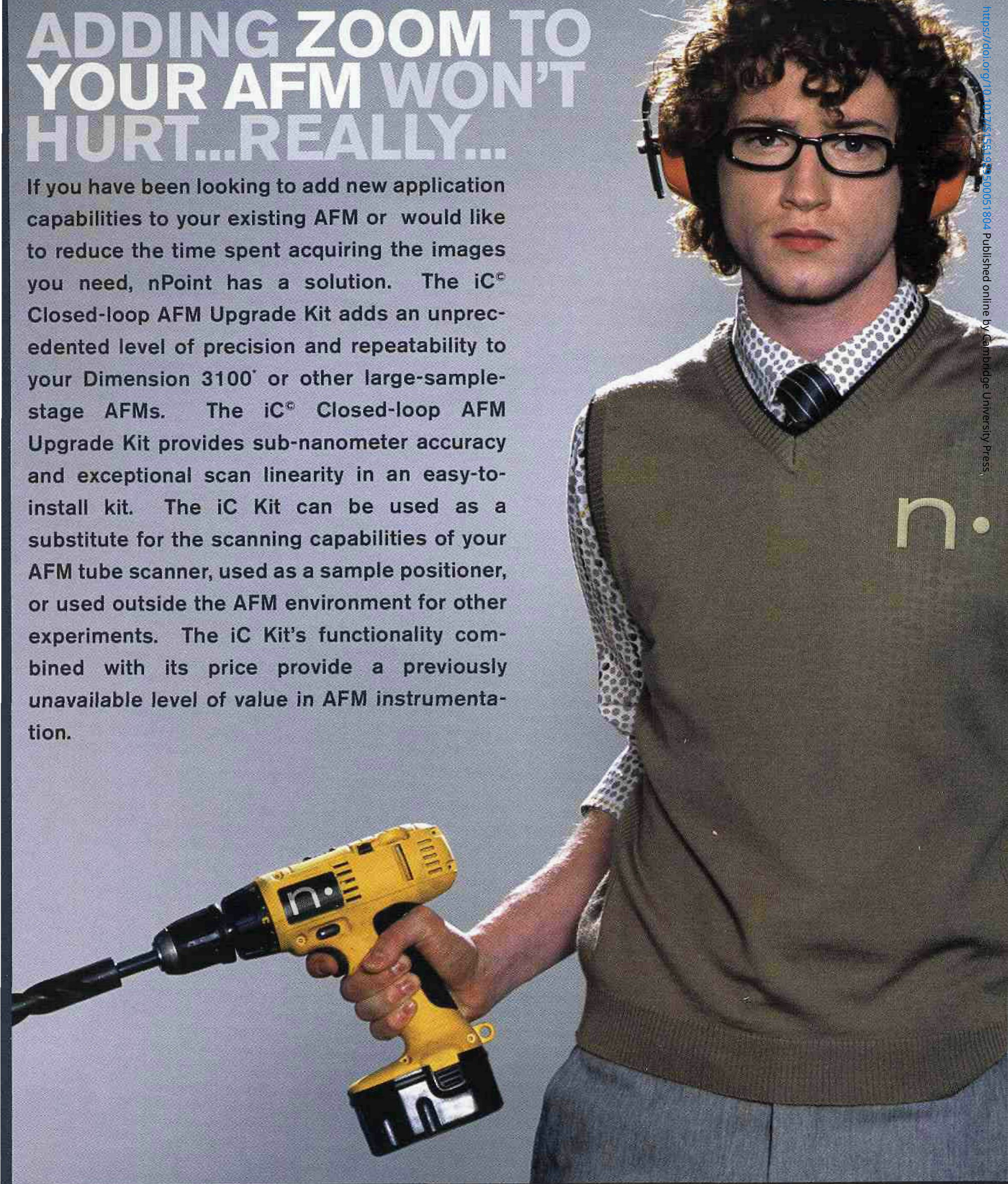

THE BEST VALUE INATOMICFORCE MICROSCOPY
FOR MORE INFORMATION: www.npoint.com sales@npoint.com (608) 204.8758

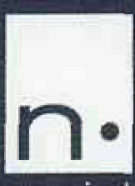

npoint 
tinuous scanning for over $15 \mathrm{~h}, \mathrm{a} \sim 10$-nm-diameter MWNT showed no detectable degradation in lateral resolution of $8-10 \mathrm{~nm}$ silicon nitride grains. The probe length also did not change with time. This was in contrast with the rapid wear of a silicon probe tip. The thinner SWNT probe tips were capable of lateral resolution as small as $2 \mathrm{~nm}$. Similar results were found in the degredation studies performed by Larsen et al. [5].

Advanced Lithography

Semiconductor structures start out as a photoresist pattern on a thin film that will be subsequently patterned by a plasma etching process. The pattern is formed by first exposing the photoresist layer to a deep ultraviolet, $X$-ray, or electron-beam pattern and then chemically developing the latent image. As the widths of the resist pattern shrink below $100 \mathrm{~nm}$, the roughness of the developed pattern edge becomes an increasingly large fraction of the allowed critical dimension tolerance budget. Many factors may contribute to line edge roughness (LER), including aerial image fluctuations, resist material, exposure parameters, and the resist development process. AFMs with CNT probe tips offer the resolution needed to non-destructively and quantitatively study the effects of photomask imaging parameters on LER [6,7]. Fig-

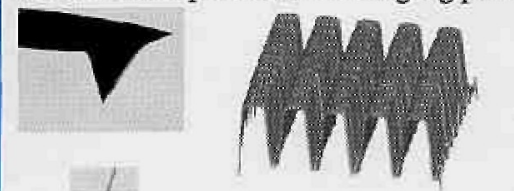

ure 4 shows a 3 -D image of a 0.5 - $\mu \mathrm{m}$ line/space photoresist pattern that resolve line edge roughness. Figure 5 shows the results of an investigation of the effect of aerial image contrast on LER using CNT AFM measurements of LER.

$0.5 \mathrm{rm}$ line and space patte

Figure 4. Right: $3-D$ CNT AFM image of $0.5-\mu m$ line/space photoresist pattern that resolves line edge roughness. Left: Transmission electron microscope images of the nanotube probe tip. [9].

\section{Biological Research}

One of the advantages of an AFM is that it can operate in a liquid medium, making it possible to examine biological specimens such as proteins, cellular structures, and DNA in a "natural" environment [10]. Carbon nanotube tips extend the resolution of AFMs used in this manner [11]. An extension of this technique is an operating mode where the AFM cantilever is driven with an oscillating magnetic field, minimizing the effect of acoustic resonance from the cantilever holder and the body, increasing the signal/noise, and reducing the required vibration amplitude [12].

CNTs have other advantages for biological studies. They are flexible and are less likely to damage fragile entities. In addition, carbon
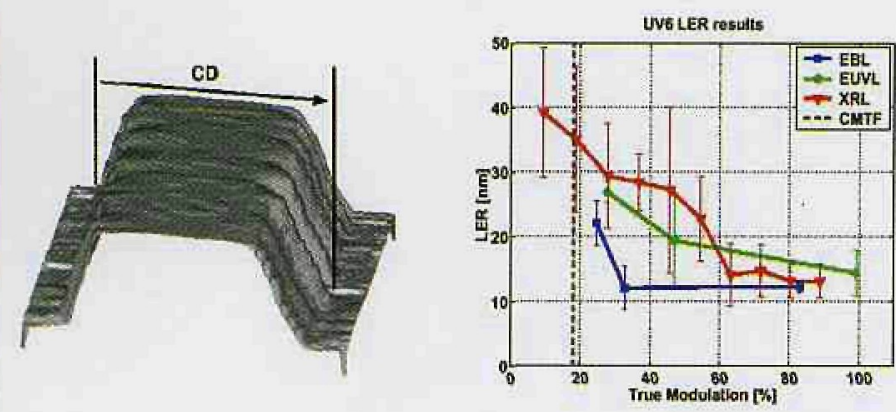

Figure 5. Left: Typical CNT AFM image of photoresist pattern. Right: The relation between line edge roughness (LER) and image contrast $(\%$ modulation) for various exposure conditions. LER decreases as image contrast increases up to a certain threshold. EBL, EUVL, and XRL refer to electron beam, extreme ultraviolet, and $x$-ray lithography respectively. CMTF (critical modulation transfer function) is the minimum intensity swing (contrast) required to print lines and spaces [6]. nanotubes can bind reversibly with chemically reactive functional groups that can sense target molecule $[13,14]$. For example a carboxylic acid (-COOH) group, when attached to a CNT tip, can "see" amide (-NH2) groups on protein molecules.

\section{Commercial Nanotube AFM Tips}

In much of the work described above the researchers had to prepare their own CNT-tipped AFM probes. Today, CNT AFM probes are available in configurations suitable for a broad range of applications. Table I lists the characteristics of typical probe configurations. CNT AFM probes provide extremely long life and high resolution for imaging high aspect-ratio samples, and samples that require gentle probe-sample interaction.

Table I. Characteristics of Carbon Nanotube Tips

\begin{tabular}{|l|c|c|c|}
\hline Characteristic & $\begin{array}{c}\text { A. High Aspect Ratio/ } \\
\text { High Specification }\end{array}$ & $\begin{array}{c}\text { B. High Aspect } \\
\text { Ratio }\end{array}$ & C. High Resolution \\
\hline Tube Angle & $\pm 5^{\circ}$ & $\pm 15^{\circ}$ & \\
\hline Diameter & $10-40 \mathrm{~nm} ;$ & $10-40 \mathrm{~nm}$ & $5-20 \mathrm{~nm}$ \\
\hline Length & $500-2000 \mathrm{~nm}$ & $500-2000 \mathrm{~nm}$ & $<500 \mathrm{~nm}$ \\
\hline
\end{tabular}

Typical CNTs are produced by attaching multi-wall carbon nanotubes onto commercial silicon (Si) tips. They function best scanning in intermittent contact mode. They are available in three types with each configuration available on standard $\mathrm{Si}$ and platinum/iridium (Pt/Ir) coated Si probes. The combinations of configurations and coatings allow optimization across a broad array of applications depending on the dimensions and alignment of the nanotube tip. The silicon cantilever on which the nanotubes are mounted has a typical spring constant of $50 \mathrm{~N} / \mathrm{m}$ and resonant frequency of $300 \mathrm{kHz}$. A Pt/Ir coated Si tip has a typical cantilever spring constant of $5 \mathrm{~N} / \mathrm{m}$ and a resonant frequency of $80 \mathrm{kHz}$.

Figure 6 shows SEM images of typical examples of each of the tip types. SEM images for each specific tip are provided with the tip. Type A. High Aspect Ratio/High Specification Tips

These tips are inspected in the scanning electron microscope from two directions to ensure that they are within $\pm 5^{\circ}$ of perpendicularity to the sample. Lengths and diameters vary with typical lengths being $500-2000 \mathrm{~nm}$ and typical diameters $10-40 \mathrm{~nm}$. A typical application is the imaging of high aspect ratio features such as narrow, deep trenches that have sharp (nearly perpendicular) edges.

Type B. High Aspect Ratio Tips

These tips are inspected in the scanning electron microscope from one direction. Their alignment is within $\pm 15^{\circ}$ from perpendicular to the sample in one direction. Typical lengths are 500-2000 $\mathrm{nm}$ and typical diameters $10-40 \mathrm{~nm}$. These tips are used in similar applications like the ones mentioned above, but their alignment with respect to the sample is not guaranteed to better than 15 degrees. Type C. High Resolution Tips

These tips have a multi-wall nanotube with diameter of 5-20 nm and a length generally shorter than $500 \mathrm{~nm}$. Such dimensions make these tips well suited for applications where high resolution, more than high aspect ratio, is desirable. Due to the short length of these tips, tip angle is not a critical. Typical applications include inspection of biological samples and topography produced by advanced lithography that has high aspect ratio features with critical dimensions on the order of a few tens of nanometers.

\section{The Future}

The potential benefits of carbon nanotube tips are yet to be fully explored. As the advantages of CNT AFM tips become widely recognized and experience is gained with their properties they will become an even more valuable addition to the AFM arsenal 

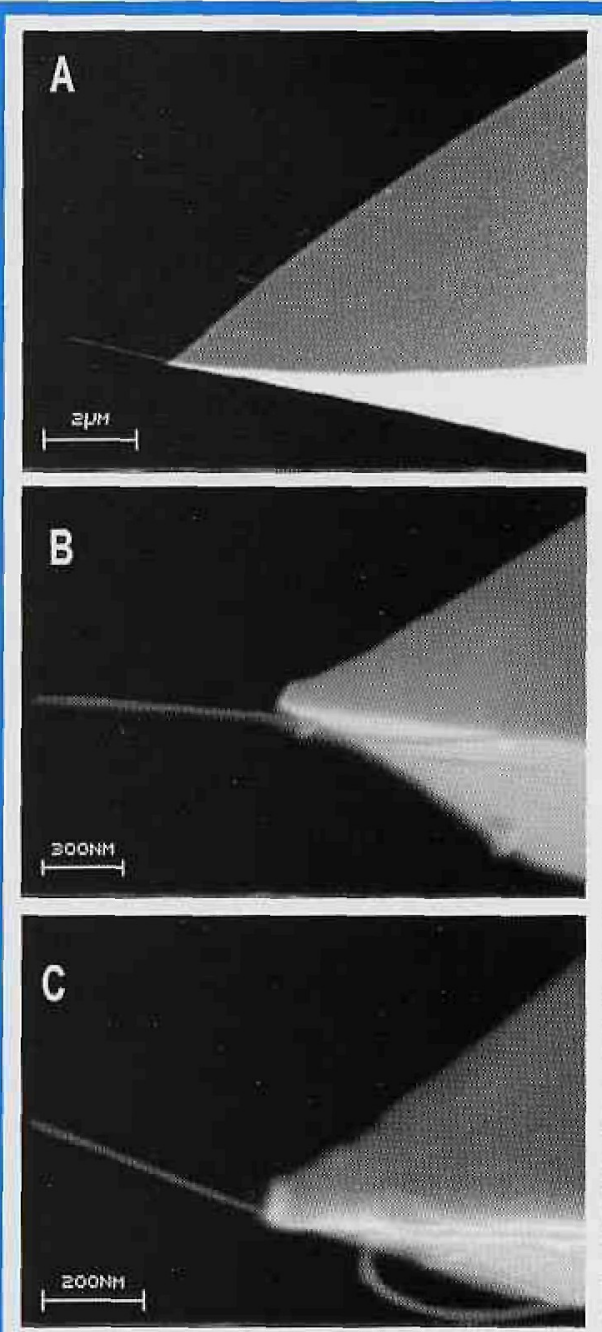

Figute 6. Scanning electron micrographs of typical CNT probe tips on silicon pyramids on silicon cantilevers. See Table I for tip characteristics.

$(2000)$

4.C. V.Nguyen, et al., "Carbon nanotube tip probes: Stability and lateral resolution in scanning probe microscopy and application to surface science in semiconductors," Nanotechnology 12, pp. 363-367 (2001)

5. T. Larsen, K. Moloni, F. Flack, M.A. Eriksson, M.G. Lagally, and C.T. Black "Comparison of wear characteristics of etched-silicon and carbon nanotube atomic-force microscopy probes," APL 80(11),pp 1996-1998 (2002).

6. J. Shin, G. Han, Y. Ma, K. Moloni, and F. Cerrina, "Resist edge line roughness and aerial image contrast," J. Vac. Sci, Technol. B 19(5), pp. 2890-2895 (Nov/Dec 2001).

7. Y. Ma, I. Shin, and F. Cerrina, "Line edge roughness and photoresist percolation development model," J. Vac. Sci. Technol. B 21(1), pp. 112-117 (Jan/Feb 2003).

8. J. Shin and F. Cerrina, "Carbon Nanotubes Application to Sub-100-nm Pattern Metrology" Semiconductor Research Corp. (unpublished), 17 July 2001.

9. J. Shin, G. Han, E. Cerrina, and K. Moloni, "Resist line-edge roughness and aerial image contrast," Semiconductor Research Corp. (unpublished), 17 July 2001.

10. R. Lewis, "From Buckyballs to Nanotubes: Carbon nanotube atomic force microscopy probes Alzheimer's disease," The Scientist 15(4), p. 8 (19 Feb 2001).

11. S.S. Wong, et al, Carbon nanotube tips: High-resolution probes for imaging biological systems," J. Am. Chem. Soc. 120, pp. 603-604 (1998).

12. J. Li and A. Cassell, "Carbon Nanotube Tips for MAC Mode" AFM Measurements in Liquids," Application Note, Molecular Imaging, Inc., Phoenix AZ.

13.S.S. Wong, E. Joselevich, A. T.Woolley, C. L. Cheung, and C.M. Lieber, "Covalently functionalized nanotubes as nanometre-sized probes in chemistry and biology," Nature 394, pp. 52-55 (1998).

14. P. J. Boul, et al., "Reversible Sidewall Functionalization of Buckytubes," Chem. Phys. Lett. 310, pp. 367-372 (1999).

15. G. Zhang, X. Jiang, and E. Wang, Science 300, pp. 472-474 (18 April 2003).

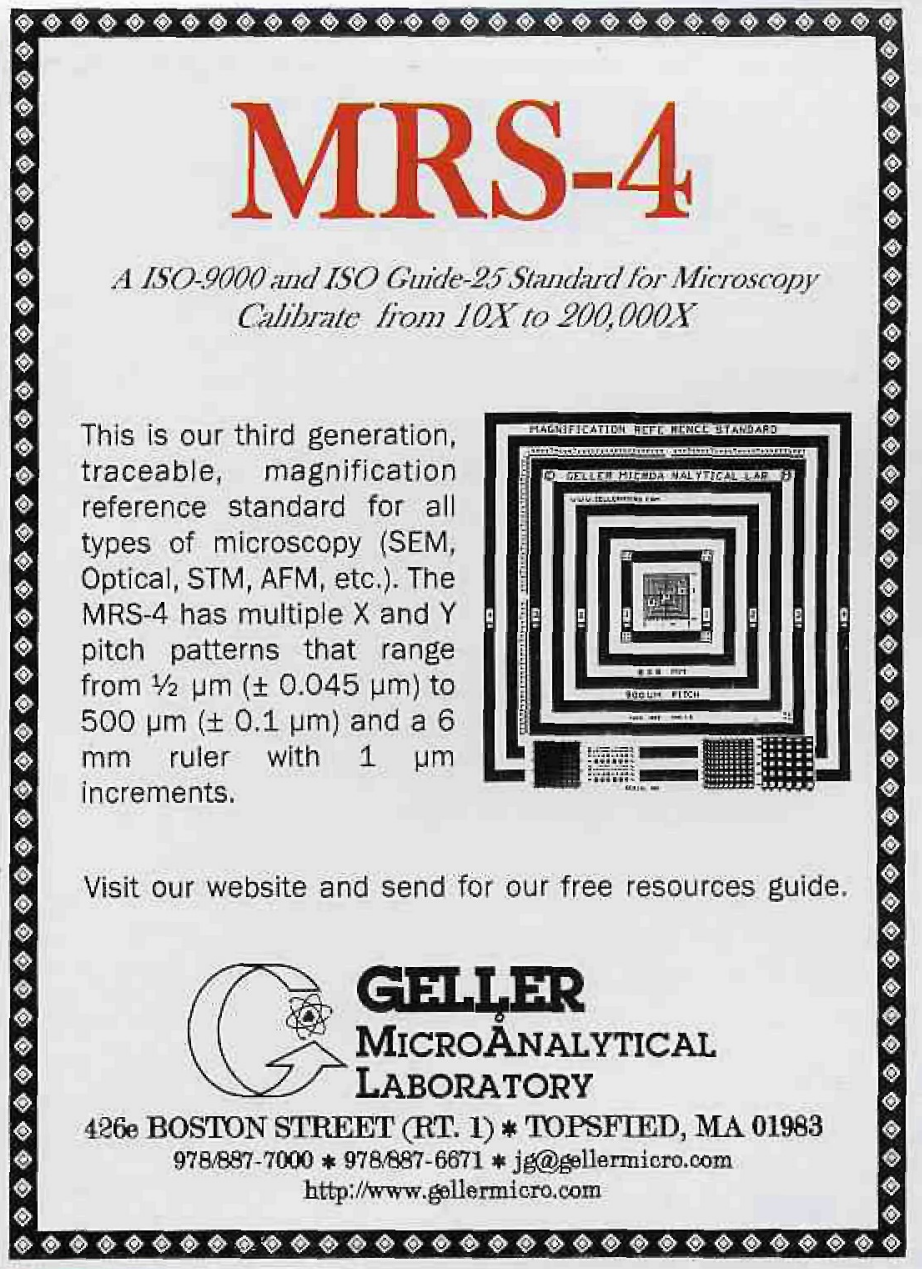

\section{Director \\ Cell Imaging Core Facility}

Fox Chase Cancer Center has an opening to lead its facility which contains state-of-the-art computer and light microscopy equipment, including a Bio-Rad Radiance 2000 Laser Scanning Confocal Microscope, an optical imaging system with upright and inverted Nikon microscopes, and an automated fluorescent dissecting microscope. Day to day responsibilities include the training of new users, maintenance and operation of the imaging systems, archiving of images on Unix and PC based computer systems, as well as the independent design and implementation of imaging-based experiments. This facility supports up to 70 investigators and is supported by an $\mathrm{NCl}$ core grant. The ideal candidate will possess a B.Sc., MS or Ph.D. degree and possess experience with confocal and wide field microscopy as well as state-ofthe-art imaging software. Salary will be commensurate with training and experience. Send $\mathrm{CV}$ and at least two letters of recommendation to: TR-Director, c/o Human Resources, Fox Chase Cancer Center, 333 Cottman Ave, Philadelphia, PA 19111-2497. www.fccc.edu

\section{Equal Opportunity Employer}

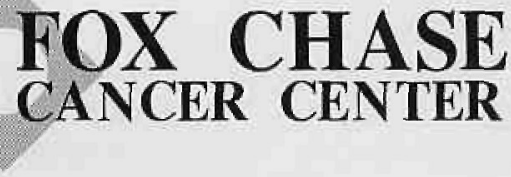

